\title{
Astrocyte senescence contributes to cognitive decline
}

\author{
Tamas Csipo • Agnes Lipecz • Nicole M. Ashpole • \\ Priya Balasubramanian - Stefano Tarantini (D)
}

Received: 18 November 2019 / Accepted: 19 November 2019 / Published online: 26 November 2019

(C) American Aging Association 2019

Aging is the main risk factor for the most pervasive diseases of developed countries including age-related cognitive decline and neurodegeneration, which afflict a continuously growing number of individuals worldwide (Franceschi et al. 2018). Aging can be broadly attributed to the life-long accumulation of mutations caused by environmental stressors that lead to a progressive loss of fitness in a species. Environmental factors such as genotoxic stressors and radiation as well as the cell's own metabolic production of reactive oxygen

T. Csipo · A. Lipecz $\cdot$ P. Balasubramanian · S. Tarantini Vascular Cognitive Impairment and Neurodegeneration Program, and Reynolds Oklahoma Center on Aging, University of Oklahoma Health Sciences Center, Oklahoma City, OK, USA

T. Csipo $\cdot$ A. Lipecz $\cdot$ S. Tarantini

International Training Program in Geroscience, Doctoral School of Basic and Translational Medicine/Department of Public Health, Semmelweis University, Budapest, Hungary

A. Lipecz

Department of Ophthalmology, Josa Andras Hospital, Nyiregyhaza, Hungary

N. M. Ashpole

Department of BioMolecular Sciences, University of Mississippi School of Pharmacy, Oxford, MS, USA

S. Tarantini $(\bowtie)$

Department of Biochemistry and Molecular Biology, Center for Geroscience and Healthy Brain Aging, University of Oklahoma HSC, 975 N. E. 10th Street - BRC 1303, Oklahoma City, OK 73104, USA

e-mail: stefano-tarantini@ouhsc.edu species (ROS) can lead to a time-dependent accumulation of DNA damage (Niccoli and Partridge 2012). As the loss of genomic fidelity burden increases, cellular defense systems monitor and repair any damage and, when damage is beyond repair, enter a proliferative arrested state termed replicative senescence. Recent evidence has shown that accumulating senescent cells in tissues and organs are a major driver of the aging process and age-related disease in mammals (Childs et al. 2015). Selective removal of senescent cells in genetically engineered animals has been demonstrated to reverse or delay aspects of aging (Perrott et al. 2017; Ogrodnik et al. 2019). Senescent cells are metabolically active, but their function has been altered in comparison to their non-senescent counterparts. A major feature exhibited by senescent cells is expression of the senescenceassociated secretory phenotype (SASP) (Watanabe et al. 2017). Mediators secreted from senescence cells can act in a paracrine manner on neighboring cells to induce senescence-like phenotypic and functional changes and thereby contribute to organ dysfunction and pathogenesis of age-related diseases, driving organismal aging. The accumulation of cellular stress also provokes adaptive and plastic transcriptional responses, which are partly orchestrated by alternative splicing (Mastrangelo et al. 2012). Alternative splicing is a critical mechanism for expanding regulatory and functional diversity from a limited number of genes, and is particularly complex in the mammalian brain (Lin et al. 2016). Alternative splicing is mediated by the competitive binding of a series of splicing activators and inhibitors 
to splicing enhancer and silencer sequences around the splice sites to determine the likelihood for each splice site to be used (Fu and Ares Jr. 2014; Smith and Valcarcel 2000).

It is estimated that $50 \%$ of the human brain mass is composed of astrocytes, which play a fundamental role in multiple aspects involved in the maintenance of brain cerebrovascular health (Jakel and Dimou 2017). Recent evidence has suggested that the accumulation of senescent astrocytes may drive neurodegenerative disorders (Di Malta et al. 2012) and recent work shows that clearance of senescent glial cells may help with by preventing the accumulation of Tau aggregates and prevent cognitive decline in mouse models (Bussian et al. 2018; Baker and Petersen 2018). Pre-clinical studies have shown that pharmacological impairment of astrocytic function recapitulates cognitive deficits that are observed in old age (Tarantini et al. 2017a). It is also noteworthy that irradiation-induced accumulation of senescent cells is associated with neurovascular dysfunction in animal models (Ungvari et al. 2017). Astrocyte senescence has also been recently implicated in the pathogenesis of Alzheimer's disease (Bhat et al. 2012; Garwood et al. 2017) and Parkinson's disease (Chinta et al. 2018). Yet, the role played by alternative splicing on the astrocytic senescence phenotype, and its involvement in the progression of age-related neurodegenerative diseases, has remained elusive.

It is appealing to conjecture that DNA damageinduced dysregulation in alternative splicing mechanisms in senescence would play a pivotal role in the process leading to the senescence-associated phenotype of astrocytes and the consequent loss of cognitive function. In support of this, recent evidence has shown that human aging is characterized by focused changes in gene expression and deregulation of alternative splicing (Harries et al. 2011) and that age-related associations between splicing factor expression and cellular senescence are present in in vitro models (Holly et al. 2013), human aging (Lee et al. 2019), and in long-lived mice (Lee et al. 2016). Emerging evidence suggests moderation of splicing factor levels is associated with reversal of cellular senescence in human primary fibroblasts (Latorre et al. 2017). Recent studies have demonstrated dysregulation of splicing mechanisms in Alzheimer's disease (Wong 2013) and that global dysregulation of splicing is characteristic of several neurodegenerative conditions such as Huntington's disease (Lin et al. 2016), frontotemporal lobar dementia (Gao et al.
2017), and Parkinson's disease (Soreq et al. 2012). Though these existent findings would predict that differential expression of splicing regulatory factors would contribute in the development of the DNA damageinduced senescent phenotype in astrocytes, no formal study has been performed to investigate altered patterns of alternative splicing in vitro in senescent astrocytes. Additionally, the relationship between some of these isoform changes detected in peripheral blood in human populations and cognitive phenotypes has not been previously described.

In an elegant paper recently published in Geroscience, Lye et al. ${ }^{28}$ identify a novel role for splicing factor expression dysfunction in the regulation of cellular senescence in astrocytes associated to the development of cognitive impairment in participants from the InCHIANTI (Ferrucci et al. 2000) population study of aging. The authors first characterized the composition of the astrocyte SASP in terms of cytokine and MMP production. Senescent astrocytes demonstrated altered levels of several key SASP proteins such as elevated IL8, GM-CSF, angiogenin, ENA78, GRO-a, MMP-3, MMP-10, and TIMP2 levels and reduced IL-10 levels (Lye et al. 2019). In their results, senescent astrocytes also displayed changes in splicing factor expression and patterns of alternative splicing. Half of the splicing factor tested in their pre-selected panel demonstrated lower expression in senescent astrocytes compared with non-senescent cells. They found dysregulation in the HNRNP splicing inhibitors and Serine-arginine (SR)rich splicing activator transcripts; also, 50\% of splicing factor transcripts measured in their panel exhibit altered expression in senescent astrocytes. Circulating level of senescence markers such as P16INK4a and functional status in humans are correlated as it is demonstrated in recent studies (Lawrence et al. 2018). To investigate the relationship between astrocyte senescence-associated transcripts and cognitive decline in a clinically relevant approach, the authors compared senescence-related transcript changes in aged primary human astrocytes with cognitive decline as assessed by changes in MMSE score between the 3rd and 4th subsequent follow-up, and in peripheral blood mRNA from individuals in the InCHIANTI study of aging. They discovered a transcriptional signature of 7 genes which associated with senescence in late passage astrocytes, as well as expressed in peripheral blood: TAU3, GFAP(A),

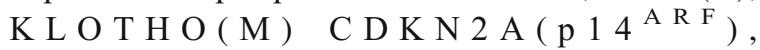
CDKN2A(p16 $\left.6^{\mathrm{INK} 4 \mathrm{~A}}\right), \mathrm{CDKN} 1 \mathrm{~A}(\mathrm{p} 21 \mathrm{~b})$, and PSEN2. 
CDKN2A (p14 ${ }^{\mathrm{ARF}}$ ) and TAU3 were positively associated with mild cognitive decline, whereas GFAP $\alpha$ was negatively associated with mild cognitive decline. Interestingly, the only association the authors found with severe cognitive decline was a negative association with TAU3. This study identifies a novel role for splicing factor expression dysfunction in the regulation of cellular senescence in astrocytes. Among the investigated candidate genes, 7 newly recognized isoforms show splicing alterations in senescent cells and concomitant expression in human peripheral blood, allowing for the discovery that TAU3, GFAPa, and CDKN2A (p14ARF) show correlation with mild cognitive decline in aging humans. These newly obtained findings suggest that age-related splicing factor changes may lead to splicing patterns for genes with roles in brain function or senescence, which may play a role in the development of cognitive decline in the human population. Astrocytes also play a key role in the regulation of cerebral blood flow by mediating neurovascular coupling responses and maintaining overall brain health. Neurovascular coupling is the term used to describe alterations in local perfusion that occur in response to changes in neuronal activity. During elevated brain activity, increased neuronal firing is sensed at the tri-partite synapse by astrocytic end-feet terminals which elicit a $\mathrm{Ca}^{2+}$ signal that travels to the end-feet enveloping the cerebral arterioles, leading to release of lipid mediators (epoxyeicosatrienoic acids [EETs] and prostaglandins), and ATP, which produce local vasodilation (Tarantini et al. 2017b; Iadecola and Nedergaard 2007). Astrogliosis, or the adoption of a stressed phenotype, is commonly observed in aged brains. Moreover, recent studies have suggested that $\sim 15 \%$ of astrocytes undergo cellular senescence (Clarke et al. 2018) in aged neurodegenerative disease states, implicating astrocytic senescence in the pathogenesis of Alzheimer's disease (Bhat et al. 2012; Garwood et al. 2017) and Parkinson's disease (Chinta et al. 2018). It is plausible to speculate that impaired mediation of neurovascular coupling responses due to the age-related increase in senescent astrocyte burden in the brain would result in impaired production of astrocyte-derived vasoactive mediators, resulting in impaired cognitive function and vascular dementia-related pathologies. Many more aspects of central nervous system physiology are mediated by astrocytes. Some of the central roles in which astrocytes are involved include ion balance, metabolism, $\mathrm{pH}$ regulation, neurotransmitter homeostasis, neurogenesis, synaptic plasticity, operation of lymphatic system, glycogen synthesis and storage, regulation of energy balance, and chemosensing (Verkhratsky and Nedergaard 2018; Verkhratsky et al. 2019a, b). It is then clear that astrocytic functional integrity is absolutely essential for brain homeostasis and overall health. In addition to the several functions performed by astrocytes control over blood-brain barrier (BBB) integrity is of particular importance for brain health and maintenance of cognitive function. The BBB is largely comprised of endothelial cells (ECs), pericytes, and astrocytes which together form an essential regulatory barrier between the neural interface and the brain vasculature. Healthy astrocytes provide essential secreted factors that lead to the adequate association between the cells of the BBB and the formation of strong tight junctions (Cabezas et al. 2014). In age-related neurological pathophysiological disorders, such as Alzheimer and Parkinson's diseases, a disruption of the BBB takes place, involving an increase in barrier permeability and phenotypical changes in both the ECs and astrocytes (Haseloff et al. 2005; Daneman 2012). Astrocyte senescence can therefore be hypothesized as an important age-related contributor to the decrease in barrier function which may result in neuroinflammation and decreased cognitive integrity and pathology observed in aging. The existing evidence presented suggests that future studies should continue to explore the functional consequences of astrocyte senescence and its relationship to declining cognitive function in aging with particular emphasis on the role of senescent astrocytes in the impairment of neurovascular coupling responses and the increase in blood-brain barrier permeability. It will still be also of great interest to determine whether similar transcriptional changes observed by Lye et al. (Lye et al. 2019) may also be occurring in other important proliferative brain cell types in the neurovascular unit such as endothelial cells. In conclusion, this work by Lye and coworkers provides an exciting step toward understanding how astrocyte senescence contributes to cognitive decline. Beyond the new questions that are raised, these data, combined with the potential new role of alterative splicing in association with cellular senescence and cognitive impairment, provide compelling evidence of the importance of DNA damage-induced processes in the age-related loss of cognitive integrity. A deeper understanding of mechanisms associated with cellular senescence in aging may provide exciting new opportunities for the development of therapies to prevent cognitive impairment in the elderly. 
Funding information This work was supported by grants from the American Heart Association (to ST), the NIA-supported Geroscience Training Program in Oklahoma (T32AG052363), the NIA-supported Oklahoma Nathan Shock Center, and the NIGMS supported Center of Biomedical Research Excellence (CoBRE).

\section{Compliance with ethical standards}

Conflict of interest The authors declare that they have no conflict of interest.

\section{References}

Baker DJ, Petersen RC (2018) Cellular senescence in brain aging and neurodegenerative diseases: evidence and perspectives. J Clin Invest 128:1208-1216

Bhat R, Crowe EP, Bitto A, Moh M, Katsetos CD, Garcia FU, Johnson FB, Trojanowski JQ, Sell C, Torres C (2012) Astrocyte senescence as a component of Alzheimer's disease. PLoS One 7:e45069

Bussian TJ, Aziz A, Meyer CF, Swenson BL, van Deursen JM, Baker DJ (2018) Clearance of senescent glial cells prevents tau-dependent pathology and cognitive decline. Nature. 562: 578-582

Cabezas R, Avila M, Gonzalez J, El-Bacha RS, Baez E, GarciaSegura LM, Jurado Coronel JC, Capani F, Cardona-Gomez GP, Barreto GE (2014) Astrocytic modulation of blood brain barrier: perspectives on Parkinson's disease. Front Cell Neurosci 8:211

Childs BG, Durik M, Baker DJ, van Deursen JM (2015) Cellular senescence in aging and age-related disease: from mechanisms to therapy. Nat Med 21:1424-1435

Chinta SJ, Woods G, Demaria M, Rane A, Zou Y, McQuade A, Rajagopalan S, Limbad C, Madden DT, Campisi J, Andersen JK (2018) Cellular senescence is induced by the environmental neurotoxin paraquat and contributes to neuropathology linked to Parkinson's disease. Cell Rep 22:930-940

Clarke LE, Liddelow SA, Chakraborty C, Munch AE, Heiman M, Barres BA (2018) Normal aging induces A1-like astrocyte reactivity. Proc Natl Acad Sci U S A 115:E1896-E1905

Daneman R (2012) The blood-brain barrier in health and disease. Ann Neurol 72:648-672

Di Malta C, Fryer JD, Settembre C, Ballabio A (2012) Astrocyte dysfunction triggers neurodegeneration in a lysosomal storage disorder. Proc Natl Acad Sci U S A 109:E2334-E2342

Ferrucci L, Bandinelli S, Benvenuti E, Di Iorio A, Macchi C, Harris TB, Guralnik JM (2000) Subsystems contributing to the decline in ability to walk: bridging the gap between epidemiology and geriatric practice in the InCHIANTI study. J Am Geriatr Soc 48:1618-1625

Franceschi C, Garagnani P, Morsiani C, Conte M, Santoro A, Grignolio A, Monti D, Capri M, Salvioli S (2018) The continuum of aging and age-related diseases: common mechanisms but different rates. Front Med (Lausanne) 5:61
Fu XD, Ares M Jr (2014) Context-dependent control of alternative splicing by RNA-binding proteins. Nat Rev Genet 15:689701

Gao FB, Almeida S, Lopez-Gonzalez R (2017) Dysregulated molecular pathways in amyotrophic lateral sclerosisfrontotemporal dementia spectrum disorder. EMBO J 36: 2931-2950

Garwood CJ, Ratcliffe LE, Simpson JE, Heath PR, Ince PG, Wharton SB (2017) Review: astrocytes in Alzheimer's disease and other age-associated dementias: a supporting player with a central role. Neuropathol Appl Neurobiol 43:281-298

Harries LW, Hernandez D, Henley W, Wood AR, Holly AC, Bradley-Smith RM, Yaghootkar H, Dutta A, Murray A, Frayling TM, Guralnik JM, Bandinelli S, Singleton A, Ferrucci L, Melzer D (2011) Human aging is characterized by focused changes in gene expression and deregulation of alternative splicing. Aging Cell 10:868-878

Haseloff RF, Blasig IE, Bauer HC, Bauer H (2005) In search of the astrocytic factor(s) modulating blood-brain barrier functions in brain capillary endothelial cells in vitro. Cell Mol Neurobiol 25:25-39

Holly AC, Melzer D, Pilling LC, Fellows AC, Tanaka T, Ferrucci L, Harries LW (2013) Changes in splicing factor expression are associated with advancing age in man. Mech Ageing Dev 134:356-366

Iadecola C, Nedergaard M (2007) Glial regulation of the cerebral microvasculature. Nat Neurosci 10:1369-1376

Jakel S, Dimou L (2017) Glial cells and their function in the adult brain: a journey through the history of their ablation. Front Cell Neurosci 11:24

Latorre E, Birar VC, Sheerin AN, Jeynes JCC, Hooper A, Dawe HR, Melzer D, Cox LS, Faragher RGA, Ostler EL, Harries LW (2017) Small molecule modulation of splicing factor expression is associated with rescue from cellular senescence. BMC Cell Biol 18:31

Lawrence I, Bene M, Nacarelli T, Azar A, Cohen JZ, Torres C, Johannes G, Sell C (2018) Correlations between age, functional status, and the senescence-associated proteins HMGB2 and p16(INK4a). Geroscience. 40:193-199

Lee BP, Pilling LC, Emond F, Flurkey K, Harrison DE, Yuan R, Peters LL, Kuchel GA, Ferrucci L, Melzer D, Harries LW (2016) Changes in the expression of splicing factor transcripts and variations in alternative splicing are associated with lifespan in mice and humans. Aging Cell 15:903-913

Lee BP, Pilling LC, Bandinelli S, Ferrucci L, Melzer D, Harries LW (2019) The transcript expression levels of HNRNPM, HNRNPA0 and AKAP17A splicing factors may be predictively associated with ageing phenotypes in human peripheral blood. Biogerontology. 20:649-663

Lin L, Park JW, Ramachandran S, Zhang Y, Tseng YT, Shen S, Waldvogel HJ, Curtis MA, Faull RL, Troncoso JC, Pletnikova O, Ross CA, Davidson BL, Xing Y (2016) Transcriptome sequencing reveals aberrant alternative splicing in Huntington's disease. Hum Mol Genet 25:3454-3466

Lye JJ, Latorre E, Lee BP, Bandinelli S, Holley JE, Gutowski NJ, Ferrucci L, Harries LW (2019) Astrocyte senescence may drive alterations in GFAPalpha, CDKN2A p14(ARF), and TAU3 transcript expression and contribute to cognitive decline. Geroscience. 
Mastrangelo AM, Marone D, Laido G, De Leonardis AM, De Vita P (2012) Alternative splicing: enhancing ability to cope with stress via transcriptome plasticity. Plant Sci 185-186:40-49

Niccoli T, Partridge L (2012) Ageing as a risk factor for disease. Curr Biol 22:R741-R752

Ogrodnik M, Zhu Y, Langhi LGP, Tchkonia T, Kruger P, Fielder E, Victorelli S, Ruswhandi RA, Giorgadze N, Pirtskhalava T, Podgorni O, Enikolopov G, Johnson KO, Xu M, Inman C, Palmer AK, Schafer M, Weigl M, Ikeno Y, Burns TC, Passos JF, von Zglinicki T, Kirkland JL, Jurk D (2019) Obesityinduced cellular senescence drives anxiety and impairs neurogenesis. Cell Metab 29:1233

Perrott KM, Wiley CD, Desprez PY, Campisi J (2017) Apigenin suppresses the senescence-associated secretory phenotype and paracrine effects on breast cancer cells. Geroscience. 39:161-173

Smith CW, Valcarcel J (2000) Alternative pre-mRNA splicing: the logic of combinatorial control. Trends Biochem Sci 25:381388

Soreq L, Bergman H, Israel Z, Soreq H (2012) Exon arrays reveal alternative splicing aberrations in Parkinson's disease leukocytes. Neurodegener Dis 10:203-206

Tarantini S, Yabluchanksiy A, Fulop GA, Hertelendy P, ValcarcelAres MN, Kiss T, Bagwell JM, O'Connor D, Farkas E, Sorond F, Csiszar A, Ungvari Z (2017a) Pharmacologically induced impairment of neurovascular coupling responses alters gait coordination in mice. Geroscience. 39:601-614

Tarantini S, Tran CHT, Gordon GR, Ungvari Z, Csiszar A (2017b) Impaired neurovascular coupling in aging and Alzheimer's disease: contribution of astrocyte dysfunction and endothelial impairment to cognitive decline. Exp Gerontol 94:52-58

Ungvari Z, Tarantini S, Hertelendy P, Valcarcel-Ares MN, Fulop GA, Logan S, Kiss T, Farkas E, Csiszar A, Yabluchanskiy A (2017) Cerebromicrovascular dysfunction predicts cognitive decline and gait abnormalities in a mouse model of whole brain irradiation-induced accelerated brain senescence. Geroscience. 39:33-42

Verkhratsky A, Nedergaard M (2018) Physiology of astroglia. Physiol Rev 98:239-389

Verkhratsky A, Zorec R, Rodriguez-Arellano JJ, Parpura V (2019a) Neuroglia in ageing. Adv Exp Med Biol 1175:181197

Verkhratsky A, Ho MS, Vardjan N, Zorec R, Parpura V (2019b) General pathophysiology of astroglia. Adv Exp Med Biol 1175:149-179

Watanabe S, Kawamoto S, Ohtani N, Hara E (2017) Impact of senescence-associated secretory phenotype and its potential as a therapeutic target for senescence-associated diseases. Cancer Sci 108:563-569

Wong J (2013) Altered expression of RNA splicing proteins in Alzheimer's disease patients: evidence from two microarray studies. Dement Geriatr Cogn Dis Extra 3:74-85

Publisher's note Springer Nature remains neutral with regard to jurisdictional claims in published maps and institutional affiliations. 